\title{
Sobre a Dinâmica de Partículas Carregadas em Campos Elétrico e Magnético
}

\author{
On Dynamic of charged particles in Electric and Magnetic fields
}

\author{
Marciano L. Medeiros ${ }^{1}$, Alan C. Santos ${ }^{* 2}$ \\ ${ }^{1}$ Departamento de Física, Universidade Regional do Cariri, Av. Leão Sampaio, 107, Triângulo, 63041-082, Juazeiro do \\ Norte, CE, Brasil \\ ${ }^{2}$ Instituto de Física, Universidade Federal Fluminense, Av. Gal. Milton Tavares de Souza s/n, Gragoatá, 24210-346, \\ Niterói, RJ, Brazil
}

Recebido em 15 de Agosto, 2016. Aceito em 22 de Agosto, 2016.

\begin{abstract}
Um desenvolvimento didático para determinar a solução da equação de movimento para uma partícula carregada imersa em uma região na presença de campos elétrico e magnéticos estáticos e homogêneos genéricos é proposto. Nossa proposta tem como alicerce a vantagem de, utilizando as propriedades da transformada de Laplace, podermos mapear um sistema de equações diferenciais não-homogêneas no problema simples de encontrar as soluções de um sistema linear de equações de primeiro grau. A partir da solução mais geral possível para o sistema, estudamos alguns casos particulares e recuperamos de forma simples alguns resultados já existentes na literatura. A fim de motivar nosso estudo, partimos do Teorema de Ehrenfest e discutimos como os resultados obtidos para o caso clássico podem ser interpretados na sua versão quântica.
\end{abstract}

Palavras-chave: movimento de cargas, campos elétrico e magnético, trajetória, análogo quântico.

A didactic development to determinate the solution of the motion equations for a charged particle under influence of electric and magnetic static and homogeneous fields is proposed. Our proposed uses the advantages and proprieties of the Laplace's transformation, to map a system of $\mathrm{N}$ non-homogeneous differential equations of second order in a system composed by $\mathrm{N}$ linear equations. From the solution more general for dynamics of the system, we study some particular cases to recover, of a simple way, the results present in the literature. In order to give a motivation to our study, we use the Ehrenfest's theorem and we discuss as the classical results can be interpreted in its quantum version.

Keywords: Charge moving, electric and magnetic fields, trajectory, quantum analogue.

\section{Introdução}

De forma elegante a Mecânica Newtoniana (Mecânica Clássica) permite-nos entender o comportamento de partículas que estão sujeitas a determinadas forças e condições iniciais [1,2]. Porém o tamanho de sistemas físicos impõe um limite sobre o regime de validade da Mecânica Clássica, de modo que sistemas do tamanho do átomo de Hidrogênio, por exemplo, podem apresentar comportamentos peculiares à luz da Mecânica Clássica. No entanto, há casos onde o tratamento clássico do comportamento

*Endereço de correspondência: alancs@if.uff.br. de um sistema pode nos dá informações relevantes sobre o sistema de interesse. Um exemplo é o de uma partícula de carga $q$ na presença de campos magnético $\vec{B}$ e elétrico $\vec{E}$ estáticos e homogêneos, como discutiremos aqui.

É conhecido que quando uma partícula penetra em uma região do espaço onde há campos magnético e elétrico ela pode estar sujeita a uma força, a depender de como ela penetra nessa região e da configuração dos campos $\vec{E}$ e $\vec{B}[3 \mid 5]$. Nossa motivação em determinar de forma genérica todas as possíveis trajetórias (para as mais gerais condições iniciais) se dá pelo fato de que não há, com facilidade em livros de física básica, uma determinação matema- 
ticamente simples da solução para as equações de movimento de tal sistema. Alguns livros resolvem casos particulares quando temos, por exemplo, apenas o campo $\vec{B}$ em ação, que mostraremos como obter esse resultado como um caso particular do nosso desenvolvimento. No entanto, um estudo matemático detalhado alternativo ao que faremos aqui, pode ser encontrado nas Refs. [3, 5], em outras usam-se provas intuitivas de que o comportamento deve ser este [6].

O ferramental matemático que será utilizado aqui é o método de solução de equações diferenciais ordinárias (ou sistemas delas) por meio da Transformada de Laplace. Esse método tem uma potencial aplicação para facilitar o processo de solução de problemas simples (como o que será tratado aqui) ou até mesmo de problemas mais complicados como o de determinar a solução da equação de Schrödinger para potenciais dependentes do tempo [7], bem como para discutir o comportamento temporal das soluções de tal equação para fenômenos de tunelamento [8,9]. A principal vantagem da utilização da tranformada de Laplace, aplicada a certos sistemas de equações diferenciais, é que podemos reduzir o problema de encontrar a solução de $N$ equações diferenciais não-homogêneas acopladas (isto é, a solução para uma dada função $x_{i}(t)$ desse sistema pode depender, a priori, do comportamento de todas as $N-1$ funções restantes) em um problema de determinar apenas a solução de um sistema linear de $N$ equações. Assim, usaremos tal benefício para tornar nosso desenvolvimento simples.

Nosso trabalho está dividido como segue. Na Seção 2 nós nos preocuparemos em definir bem o formalismo matemático que será usado na Seção 3. onde analisaremos a trajetória de uma partícula clássica que está sujeito a campos estáticos e homogêneos (independente do tempo $t$ e da posição $\vec{r}) \vec{E}$ e $\vec{B}$. Uma vez obtido, no tratamento clássico, a solução unívoca das equações de movimento do nosso sistema de interesse, na seção 3.2 nós estudaremos alguns casos particulares com relação ao arranjo dos campos $\vec{E}$ e $\vec{B}$ e da condições iniciais do problema (velocidade e posição da partícula no início do movimento). Por fim, na seção 4 nós mostramos como o tratamento clássico feito na seção 3 pode nos fornecer informações importantes a cerca da dinâmica de um ensemble (feixe) de partículas carregadas.

\section{Formalismo matemático}

O ferramental matemático usado para alcançar o objetivo deste presente artigo é a conhecida Transformada de Laplace. A transformada de Laplace é um caso particular de um conjunto de transformadas mais gerais ditas Transformadas Integrais. Em geral transformadas integrais de uma função $f(t)$ são definidas como 10.

$$
T[f(t)] \equiv F(\xi)=\int_{I} K(t, \xi) f(t) d t,
$$

onde $K(t, \xi)$ é denominado núcleo da transsformação e $I$ é um intervalo em $t$ que pode, ou não, ser infinito. A ideia básica de transformadas integrais é que, dado um problema, possamos definir uma transformada a partir de um núcleo $K(t, \xi)$ que mapeia o problema em um novo espaço de parâmetros $\xi$, de modo que nesse novo espaço seja possível resolver o problema original de forma simples. Ao final, deve ser possível retornar ao espaço dos parâmetros $t$, assim devemos definir a transformada de modo que exista uma transformada inversa associada a $T$ de modo que

$$
T^{-1}\{T[f(t)]\} \equiv T^{-1}\{F(\xi)\}=f(t) .
$$

Na Fig. 1 nós mostramos um esquema de cada etapa descrita acima. A importância da existência de uma transformada inversa é que a solução do problema no espaço do parâmetro $t$ só pode ser obtido, a partir da solução obtida no espaço do parâmetro $\xi$, se pudermos definir a transformada inversa $T^{-} 1[\bullet]$.

A transformada integral de nosso interesse está associado ao formalismo das Transformadas de Laplace (TL). Aqui discutiremos sobre as TL de forma

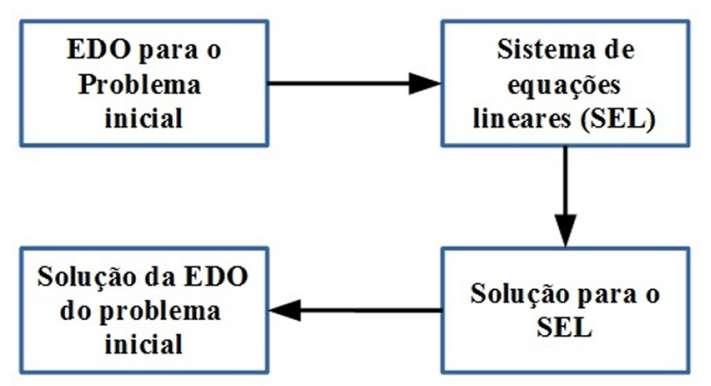

Figura 1: Diagrama do algoritmo que deve ser executado quando usa-se de transformadas integrais para solucionar algum problema. Nesse caso, nosso problema considerado é a de determinar a solução de uma EDO via transformadas de Laplace, mas o algoritmo é genérico. 
objetiva, bem como resultados e propriedades relevantes para este trabalho.

Definição 1 Seja $f:[0, \infty) \longrightarrow \mathbb{R}$ uma função integrável para todo subintervalo finito de $[0, \infty)$. A transformada de Laplace da função $f$, denotada por $\mathcal{L}[f(t)]$ ou $F(\xi)$, é definida por

$$
F(\xi)=\int_{0}^{\infty} e^{-\xi t} f(t) d t=\lim _{n \rightarrow \infty}\left[\int_{0}^{n} e^{-\xi t} f(t) d t\right],
$$

que resulta em uma função do parâmetro $\xi$.

A primeira propriedade da transformada de Laplace $\mathcal{L}$ é a linearidade, logo se $\left\{f_{i}(t)\right\}=\left\{f_{1}(t), \cdots\right.$, $\left.f_{m}(t)\right\}$ com $i=1, \cdots, m$ é um conjunto de funções cujas transformadas de Laplace existem, então

$$
\mathcal{L}\left[\sum_{i=1}^{m} a_{i} f_{i}(t)\right]=\sum_{i=1}^{m} a_{i} \mathcal{L}\left[f_{i}(t)\right]
$$

Uma aplicação notável das transformadas de Laplace é na resolução de equações diferenciais ordinárias com coeficientes constantes sujeitas à condições de contorno. Isso decorre do seguinte teorema 10

Teorema 1 Seja $f(t)$ uma função tal que sua $n$ ésima derivada com relação ao parâmetro $t$ seja integrável e dado um conjunto de condições de contorno $d^{n} f(0) / d t^{n}=c_{n}$ para todo n. Então

$$
\mathcal{L}\left[\frac{d^{n} f(t)}{d t^{n}}\right] \equiv \xi^{n} \mathcal{L}[f(t)]-\sum_{j=1}^{n} \xi^{n-j} c_{j-1}
$$

onde $\mathcal{L}[f(t)]$ é a transformada de Laplace de $f(t)$.

O resultado acima em conjunto com a propriedade de linearidade das transformadas de Laplace podem ser usados para encontrar soluções de sistemas de equações diferenciais de ordem $n$. De fato, considere um sistema de equações diferenciais nas funções $x_{i}(t)$ e com condições de contorno $d^{n} x_{i}(0) / d t^{n}=c_{n}^{i}$. A partir da Eq. (4) podemos concluir que aplicando a transformada a um sistema de ED, recaímos em um problema de encontrar soluções de sistemas de equações lineares nas variáveis $\chi_{i}(\xi)=\mathcal{L}\left[x_{i}(t)\right]$. Então, desde que o sistema de equações lineares associados ao sistema de ED nos forneça soluções integráveis $\chi_{i}(\xi)$, podemos usar a transformada de Laplace inversa e obter as soluções $x_{i}(t)$ do sistema de ED.

\section{Tratamento Clássico}

Nesta seção descreveremos o tratamento clássico da dinâmica de uma partícula sujeita a campos elétrico $\vec{E}$ e magnético $\vec{B}$. Para nosso estudo, estamos considerando o caso particular onde os campos $\vec{E}$ e $\vec{B}$ são independentes do tempo e da posição no espaço onde calculamos suas propriedades (módulo, direção e sentido), isto é, os campos $\vec{E}$ e $\vec{B}$ são estáticos e homogêneos. Para tanto, definiremos o problema a ser tratado classicamente e em seguida determinaremos a equação de movimento do sistema. Além disso nós determinamos, de forma unívoca com ajuda de condições iniciais, a solução paramétrica para a equação de movimento, onde nosso parâmetro é o tempo $t$ de evolução do sistema. Em seguida estudaremos o comportamento do sistema em casos particulares fazendo considerações relacionadas aos campos $\vec{E}$ e $\vec{B}$ e as condições iniciais do sistema.

\subsection{Solução das Equações de Movimento: Caso Geral}

Considere uma partícula de massa $m$ e carga elétrica líquida $q$ que está imersa em uma região que, no instante $t=t_{0}$, fica submetida à ação de dois campos estáticos e homogêneos, um de natureza magnética $\vec{B}$ e outro de natureza elétrica $\vec{E}$. Já é sabido que cada um destes campos, a depender das condições sobre a posição e a velocidade da partícula em $t=t_{0}$, exercerá uma força sobre a partícula, de modo que em geral a força resultante é dada pela força de Lorentz

$$
\vec{F}_{L o r}=q \vec{E}+q \vec{v} \times \vec{B} .
$$

Sem perda de generalidade nós adotamos que o nosso sistema de coordenadas tem o eixo $Z$ orientado na direção do campo $\vec{B}$ e que a configuração inicial do sistema, quando acionamos os campos estáticos, tem a forma representada pela Fig. 2

A origem do nosso sistema de coordenadas está fixada exatamente no ponto onde a partícula se localiza em $t=0$, de modo que as condições iniciais são

$$
\vec{r}(0)=(0,0,0) \text { e } \vec{v}(0)=\left(v_{1}(0), v_{2}(0), v_{3}(0)\right),
$$

que é o máximo de simplificação que conseguimos admitir sem perder a generalidade do problema. Assim, as componentes da aceleração do sistema na forma diferencial são dadas, de acordo com a $2^{\mathrm{a}}$ lei 


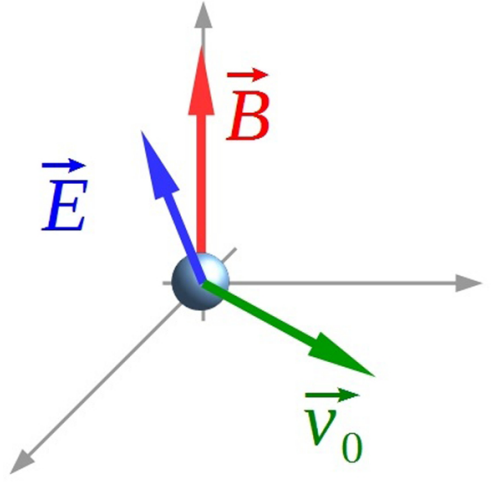

Figura 2: Configuração inicial do sistema, onde estamos adotando nosso sistema de coordenadas no ponto onde a partícula se encontra inicialmente.

de Newton, por

$$
\begin{aligned}
\frac{d^{2} x_{1}(t)}{d t^{2}} & =\frac{q}{m}\left(\frac{d x_{2}(t)}{d t} B+E_{1}\right), \\
\frac{d^{2} x_{2}(t)}{d t^{2}} & =-\frac{q}{m}\left(\frac{d x_{1}(t)}{d t} B-E_{2}\right), \\
\frac{d^{2} x_{3}(t)}{d t^{2}} & =\frac{q E_{3}}{m},
\end{aligned}
$$

onde rotulamos as componentes $x, y$ e $z$ por 1,2 e 3, respectivamente. As Eqs. (7) e (8) claramente formam um sistema de equações diferenciais acopladas, mas por outro lado vemos que a Eq. (9) está desacoplada das demais. Assim nós iniciamos por determinar a solução da Eq. (9) onde, por integração, facilmente podemos mostrar que

$$
x_{3}(t)=v_{3} t+\frac{q E_{3}}{m} \frac{t^{2}}{2},
$$

onde usaremos de agora em diante a notação $\beta_{k}(0)=$ $\beta_{k}$, é solução da Eq. (9), dando assim o comportamento da partícula ao longo do eixo $Z$ do sistema de coordenadas. Continuando, para sistema composto pelas Eqs. (7) e (8) nós usamos o método da transformada de Laplace descrito na seção 2 . Aplicando a transformada de Laplace às Eqs. (7) e (8) obtemos, de acordo com o Teorema 1 e as Eqs. (3) e (6)

$$
\begin{aligned}
& \xi^{2} \chi_{1}(\xi)-v_{1}=\frac{q}{m}\left(B \xi \chi_{2}(\xi)+\frac{E_{1}}{\xi}\right) \\
& \xi^{2} \chi_{2}(\xi)-v_{2}=-\frac{q}{m}\left(B \xi \chi_{1}(\xi)-\frac{q E_{2}}{\xi}\right)
\end{aligned}
$$

onde a partir daqui vamos definir $\omega_{0}=q B / m$ e $\gamma_{k}=q E_{k} / m$ com $k=1,2$. Para resolver o sistema acima nós primeiro resolvemos o sistema para $\chi_{1}(\xi)$ e $\chi_{2}(\xi)$ e em seguida aplicamos a transformada de Laplace inversa (5) para obtermos as soluções $x_{1}(t)$ e $x_{2}(t)$, respectivamente. $\mathrm{O}$ resultado dessa sequencia de passos é que a solução para as Eqs. (7) e (8) são, respectivamente, dadas por

$$
\begin{aligned}
x_{1}(t) & =\frac{1}{\omega_{0}^{2}}\left[A_{1}-C_{2} \cos \left(\omega_{0} t\right)\right. \\
& \left.+C_{1} \sin \left(\omega_{0} t\right)\right], \\
x_{2}(t) & =\frac{1}{\omega_{0}^{2}}\left[A_{2}+C_{1} \cos \left(\omega_{0} t\right)\right. \\
& \left.+C_{2} \sin \left(\omega_{0} t\right)\right],
\end{aligned}
$$

onde definimos as quantidades ( $\operatorname{com} k=\{1,2\})$

$$
\begin{aligned}
& A_{1}=\gamma_{2}+\left(\gamma_{1} t+v_{2}\right) \omega_{0}, \\
& A_{2}=\gamma_{1}-\left(\gamma_{2} t+v_{1}\right) \omega_{0}, \\
& C_{k}=(-1)^{k} \gamma_{k}+v_{k} \omega_{0},
\end{aligned}
$$

de forma que uma análise dimensional mostra que $\left[A_{k} / \omega_{0}^{2}\right]=\left[C_{k} / \omega_{0}^{2}\right]=[$ posição $]$. Isso nos permite concluir que a partícula descreve uma trajetória dada pelas equações paramétricas (13), (14) e (10). Das Eqs. (15), (16) e (17) podemos ver que, de fato, a configuração dos campos e a condição inicial $\vec{v}(0)$ afetam diretamente a trajetória da partícula, dando-nos uma gama de possibilidades para sua trajetória.

\subsection{Casos Particulares}

Levando em conta que a configuração dos campos e a condição inicial $\vec{v}(0)$ tem influência direta na trajetória da partícula, vamos considerar casos particulares de trajetórias que podem ser obtidas mediante certas condições inicias aplicadas às equações paramétricas (13), (14) e 10) que descrevem a trajetória geral da partícula.

\section{- Caso particular 1}

Inicialmente, vamos considerar o caso em que a partícula entra numa região contendo apenas o vetor campo magnético de tal forma que $\vec{v}(0) \perp \vec{B}$ com $\vec{v}(0)=\vec{v}_{0}=\left(v_{1}, v_{2}, 0\right)$. Neste caso, as equações paramétricas (13) e (14) tomam a seguinte forma

$$
\begin{aligned}
x_{1}(t) & =\frac{1}{\omega_{0}}\left[v_{2}\left(1-\cos \left(\omega_{0} t\right)\right)\right. \\
& \left.+v_{1} \sin \left(\omega_{0} t\right)\right], \\
x_{2}(t) & =\frac{1}{\omega_{0}}\left[v_{1}\left(1+\cos \left(\omega_{0} t\right)\right)\right. \\
& \left.+v_{2} \sin \left(\omega_{0} t\right)\right]
\end{aligned}
$$


que podem ser postas facilmente na forma

$$
\left(x_{1}(t)-\frac{v_{2}}{\omega_{0}}\right)^{2}+\left(x_{2}(t)-\frac{v_{1}}{\omega_{0}}\right)^{2}=\frac{\left\|\vec{v}_{0}\right\|^{2}}{\omega_{0}^{2}},
$$

que é a equação de uma circunferência de raio $R=$ $\|\vec{v}\| / \omega_{0}$ centrada no ponto $\left(v_{2} / \omega_{0}, v_{1} / \omega_{0}\right)$ e onde $\|\vec{v}\|=\left(v_{1}^{2}+v_{2}^{2}\right)^{\frac{1}{2}}$ é a norma da velocidade inicial da carga. Em outras palavras, a Eq. (20) mostra que a partícula executa um movimento circular no plano $x y$ com raio $R=\|\vec{v}\| / \omega_{0}$.

A fim de dar uma descrição mais completa do movimento, considere o vetor posição da partícula $\vec{r}(t)$ no instante $t$ como $\vec{r}(t)=x_{1}(t) \hat{\mathrm{i}}+x_{2}(t) \hat{\mathrm{j}}$, com $\hat{i}$ e $\hat{j}$ sendo versores na direção $x$ e $y$, respectivamente. Então, o vetor velocidade $\vec{v}(t)$ da partícula é dado por

$$
\vec{v}(t)=\frac{d \vec{r}(t)}{d t}=\dot{x}_{1}(t) \hat{\mathrm{i}}+\dot{x}_{2}(t) \hat{\mathrm{j}}=v_{1}(t) \hat{\mathrm{i}}+v_{2}(t) \hat{\mathrm{j}},
$$

onde

$$
\begin{aligned}
& v_{1}(t)=v_{2} \sin \left(\omega_{0} t\right)+v_{1} \cos \left(\omega_{0} t\right), \\
& v_{2}(t)=v_{2} \cos \left(\omega_{0} t\right)-v_{1} \sin \left(\omega_{0} t\right),
\end{aligned}
$$

com a norma do vetor $\vec{v}(t)$ sendo $\|\vec{v}(t)\|=\left\|\overrightarrow{v_{0}}\right\|$, para todo instante do movimento, configurando assim um movimento circular com velocidade de módulo constante. Portanto, podemos agora assumir $\omega_{0}$ como sendo a frequência angular do movimento circular que é executado pela partícula.

\section{- Caso particular 2}

Vamos considerar agora a configuração tal que a carga é lançada obliquamente às linhas de indução de $\vec{B}$, considerando $\vec{E}=\overrightarrow{0}$ e $\vec{v}_{0}=\left(v_{1}, 0, v_{3}\right)$. Nesse caso, usando as Eqs. (10), 113), 114) e explicitando $A_{1}, A_{2}, C_{1}$ e $C_{2}$ em termos das configurações dadas para $\vec{B}, \vec{E}$ e $\vec{v}_{0}$ temos:

$$
\begin{aligned}
& x_{1}(t)=\rho_{L} \sin \left(\omega_{0} t\right), \\
& x_{2}(t)=\rho_{L}\left[1+\cos \left(\omega_{0} t\right)\right] \\
& x_{3}(t)=v_{3} t,
\end{aligned}
$$

onde $\rho_{L}=m v_{1} /|q| B$ é conhecido na literatura como raio de Larmor. As Eqs. (24), (25) e (26) mostram que, nestas condições, a partícula descreve uma trajetória helicoidal equivalente à composição de um movimento circular com velocidade constante $v_{1} \mathrm{e}$ raio $\rho_{L}$ no plano $x y$ e de um movimento com velocidade constante $v_{3}$ na direção das linhas de indução.
A este movimento dar-se o nome de giração e o centro do movimento circular que, nesse caso, possui coordenadas $(x, y, z)=\left(0, \rho_{L}, 0\right)$, denomina-se centro de guia. Nesse caso, o centro de guia desloca-se com velocidade constante $v_{3}$ na direção das linhas de indução de forma que a giração é um circulo cuja equação no plano $x y$ é dada pela Eq. (20) tomando $v_{2}=0$ [11.

Considerando a velocidade $\vec{v}$ da partícula ao longo da trajetória helicoidal e definindo o ângulo de passo $\phi$ de tal forma que as componentes de $\vec{v}$ nas direções paralela e perpendicular a $\vec{B}$ sejam dadas por

$$
\begin{aligned}
& v_{3}=\|\vec{v}\| \cos (\phi), \\
& v_{2}=\|\vec{v}\| \sin (\phi),
\end{aligned}
$$

então o passo da hélice $\delta$ será definido como a distância percorrida pela partícula na direção paralela a $\vec{B}$ a cada período do movimento circular $\tau=\frac{2 \pi}{\omega_{0}}$, ou seja

$$
\delta=\tau v_{3}=\frac{2 \pi\|\vec{v}\|}{\omega_{0}} \cos (\phi) .
$$

Na Fig. 3 podemos visualizar a trajetória da partícula, onde a distância vertical entre dois pontos da trajetória (como sinalizado na curva em azul) é dado pela quantidade $\delta$ discutida acima.

\section{- Caso particular 3}

Agora vamos considerar o caso em que a partícula entra numa região na qual estão presentes os campos elétrico e magnético cruzados tais que $\vec{E}=\left(E_{1}, 0,0\right)$,

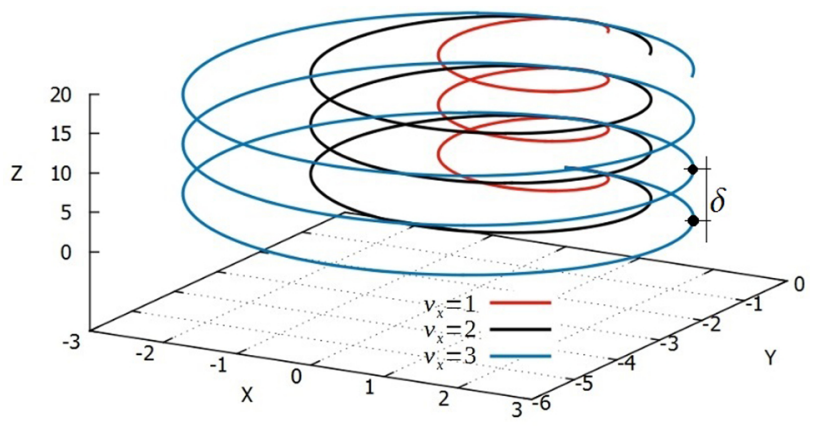

Figura 3: Trajetória helicoidal da partícula resultante da particularização no caso 2 . A escolha dos parâmetros que configuram os campos para construir o gráfico foram $\left(\gamma_{1}, \gamma_{2}, \gamma_{3}, \omega_{0}\right)=(0,0,0,1)$ (estamos adotando unidades de medidas no $\mathrm{SI}$ ). As curvas estão associadas a diferentes escolhas para a componente $x$ da velocidade inicial e fixamos a componente $z$ como a unidade. 
$\vec{B}=(0,0, B)$ e $\vec{v}_{0}=\left(v_{1}, 0,0\right)$. Com estas configurações podemos simplificar as Eqs. (13) e (14) que tomam a forma

$$
\begin{aligned}
& x_{1}(t)=\frac{1}{\omega_{0}^{2}}\left[A_{1}+C_{1} \sin \left(\omega_{0} t\right)\right], \\
& x_{2}(t)=\frac{1}{\omega_{0}^{2}}\left[A_{2}+C_{1} \cos \left(\omega_{0} t\right)\right] .
\end{aligned}
$$

Explicitando $A_{1}, A_{2}$ e $C_{1}$ em termos das configurações dadas para $\vec{E}, \vec{B}$ e $\vec{v}_{0}$ obtemos

$$
\begin{aligned}
x_{1}(t) & =\frac{q E_{1}}{m \omega_{0}^{2}}\left[\omega_{0} t+\left(\frac{\omega_{0} m v_{1}}{q E_{1}}-1\right)\right. \\
& \left.\times \sin \left(\omega_{0} t\right)\right], \\
x_{2}(t) & =\frac{1}{\omega_{0}^{2}}\left[\left(\frac{q E_{1}}{m}-\omega_{0} v_{1}\right)\right. \\
& \left.\times\left(1-\cos \left(\omega_{0} t\right)\right)\right],
\end{aligned}
$$

de forma que se $v_{1}=0$ obtemos

$$
\begin{aligned}
& x_{1}(t)=\frac{q E_{1}}{m \omega_{0}^{2}}\left[\omega_{0} t-\sin \left(\omega_{0} t\right)\right], \\
& x_{2}(t)=\frac{q E_{1}}{m \omega_{0}^{2}}\left[1-\cos \left(\omega_{0} t\right)\right],
\end{aligned}
$$

que são exatamente as equações paramétricas de uma cicloide com $t$ atuando como parâmetro.

É interessante notar aqui a existência de um efeito de focalização [12] que ocorre nesse tipo de trajetória e que obriga a partícula eletrizada a passar por uma sucessão de pontos fixos no eixo $X$, chamados de pontos focais, independentemente do valor da velocidade inicial $v_{0}$. Podemos mostrar isso tomando o conjunto de valores de $t$ que satisfaz $x_{2}=0$ na Eq. (33), que implica em ter $\cos \left(\omega_{0} t\right)=1$, de modoq que podemos construir a família de valores $t=2 \pi \lambda / \omega_{0}$ com $\lambda \in \mathbb{Z}$. Substituindo esse valor de $t$ na Eq. (32), obtemos

$$
x_{1}=2 \pi \lambda \frac{q E_{1}}{\omega_{0}^{2} m}=2 \pi \lambda \frac{m E_{1}}{q B^{2}},
$$

onde na ultima igualdade usamos que $\omega_{0}=q B / \mathrm{m}$. A equação acima fornece a posição dos pontos focais ao longo do eixo $X$, como pode ser visto na Fig. 4.

\section{O Tratamento Quântico}

Agora nós mostraremos como tratar um sistema quântico cujo seu análogo clássico foi discutido anteriormente na seção 3 . O que faremos aqui nada

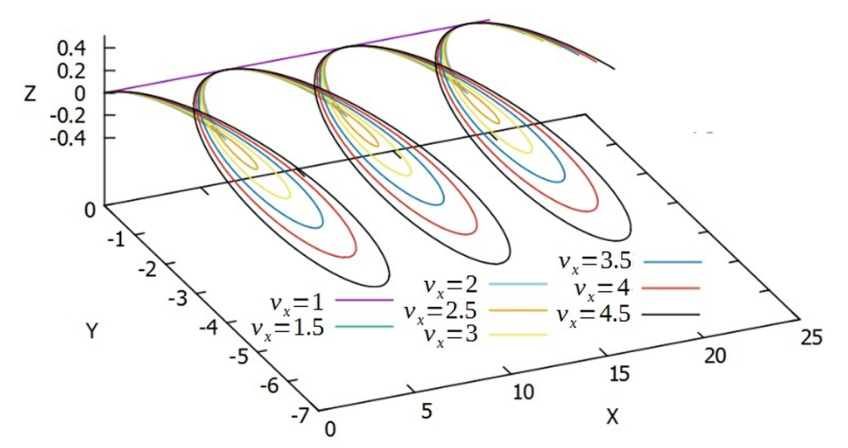

Figura 4: Trajetória da partícula como resultado da particularização no caso 3. Novamente a escolha dos parâmetros para construir o gráfico se dá como $\left(\gamma_{1}, \gamma_{2}, \gamma_{3}, \omega_{0}\right)=(1,0,0,1)$. A única componente da velocidade é na direção $x$ e plotamos o gráfico para várias escolhas deste a fim de visualizar os pontos fixos independentes da velocidade inicial. Unidades de medidas novamente no SI.

mais é do que uma aproximação na tentativa de descrever a dinâmica de um feixe de partículas cuja dinâmica de cada partícula que contitui o sistema é dada pela equação de Schrödinger. Para tal, iremos utilizar o conhecido Teorema de Ehrenfest [13. Com relação aos resultados relacionados ao Teorema de Ehrenfest, aqui nós faremos algo bastante resumido e focado no nosso objetivo, para uma discussão mais geral recomendamos a [14, onde outras referências sobre o assunto podem ser encontradas.

Nosso ponto de partida é a equação de Schrödinger dada por

$$
H(\vec{r}, t)|\psi(\vec{r}, t)\rangle=i \hbar \partial_{t}|\psi(\vec{r}, t)\rangle
$$

sendo $\partial_{t}$ o operador derivada parcial e onde $H(\vec{r}, t)$ é o Hamiltoniano que governa o sistema dado por

$$
H(\vec{r}, t)=\frac{\vec{p}^{2}}{2 m}+V(\vec{r}, t),
$$

$\operatorname{com} \vec{p}=\left(\hat{p}_{x}, \hat{p}_{y}, \hat{p}_{z}\right)$ e $\vec{r}=\left(\hat{r}_{x}, \hat{r}_{y}, \hat{r}_{z}\right)$ o operador momento e posição, respectivamente, em três dimensões e que satisfazem as relações de comutação

$$
\left[\hat{r}_{k}, \hat{p}_{m}\right]=i \hbar \delta_{k m} \mathbb{1} \quad, \quad\left[\hat{r}_{k}, \hat{r}_{m}\right]=\left[\hat{p}_{k}, \hat{p}_{m}\right]=0 .
$$

A equação de Schrödinger descreve a dinâmica de sistemas quânticos, mas existe uma maneira análoga de analisarmos a evolução de um sistema que é analisando como evoluem os valores esperados de algum observável físico. Sendo $\mathcal{O}$ um observável fisico (energia, posição, momento, etc...), então o valor esperado deste observável é definido como

$$
\langle\mathcal{O}\rangle=\langle\psi(\vec{r}, t)|\mathcal{O}| \psi(\vec{r}, t)\rangle
$$


Derivando esta equação com relação ao tempo, podemos mostrar que

$$
\frac{d}{d t}\langle\mathcal{O}\rangle=\frac{i}{\hbar}\langle[H(\vec{r}, t), \mathcal{O}]\rangle+\left\langle\partial_{t} \mathcal{O}\right\rangle,
$$

onde estamos considerando que o operador $\mathcal{O}$ não possui dependencia temporal, assim $\left\langle\partial_{t} \mathcal{O}\right\rangle=0$. Como a equação acima envolve médias de uma quantidade física, então devemos atentar para o fato de que estamos tratando de um grande número de medidas do observável $\mathcal{O}$, ou para um ensemble de partículas (feixe de elétrons, fótons, etc).

Agora deixe-nos analisar os resultados que podemos obter da Eq. (41) no caso particular de nosso interesse, ou seja, uma partícula de carga $q$ sujeito à ação de um campo eletromagnético. Já é sabido que o Hamiltoniano que governa a dinâmica desse sistema é dado por 15

$$
H(\vec{r})=2 m[\vec{p}-q \vec{A}(\vec{r}, t)]^{2}+q \phi(\vec{r}, t)
$$

onde $\vec{A}(\vec{r}, t)$ e $\phi(\vec{r}, t)$ são os potenciais vetor e escalar, respectivamente, usados para escrever os campos elétricos e magnéticos, respectivamente, sob a forma

$$
\begin{aligned}
\vec{E}(\vec{r}, t) & =-\vec{\nabla} \phi(\vec{r}, t)-\partial_{t} \vec{A}(\vec{r}, t), \\
\vec{B}(\vec{r}, t) & =\vec{\nabla} \times \vec{A}(\vec{r}, t) .
\end{aligned}
$$

Usando um algebrismo um pouco extenso e as relações de comutação na Eq. (39), bem como as equações acima, nós podemos mostrar que para o Hamiltoniano da Eq. (42) nós temos

$$
m \frac{d^{2}}{d t^{2}}\langle\vec{r}\rangle=q\left[\vec{E}+\frac{d}{d t}\langle\vec{r}\rangle \times \vec{B}\right]
$$

de modo que devemos olhar para a quantidade $\langle\vec{r}\rangle$ como $\langle\vec{r}\rangle=\langle\hat{x}\rangle \hat{\imath}+\langle\hat{y}\rangle \hat{\jmath}+\langle\hat{z}\rangle \hat{k}$, onde $\langle\hat{m}\rangle$ é o valor esperado do operador associado à $\hat{m}$-ésima coordenada da "posição"da partícula.

A Eq. (45) mostra que o comportamento do valor esperado de $\vec{r}$ é descrito por equações de movimento semelhante a Eq. (5) (como esperado do Teorema de Ehrenfest) considerando, é claro, que os campos $\vec{E}$ e $\vec{B}$ são uniformes (independentes da posição). Esse resultado nos dá a equação de movimento para um ensemble de partículas, cuja carga do ensemble é $q$, na presença de campos magnético e elétrico estáticos e uniformes, e será de maior importância para estudos mais avançados que serão desenvolvidos posteriormente.

\section{Conclusão}

A partir da transformada de Laplace estudamos o caso mais geral da dinâmica de uma partícula sujeita a interação com campos elétrico e magnético estáticos e homogêneos. Usando a definição e propriedades da transformada de Laplace, mostramos como mapear o problema de resolver um sistema de equações diferenciais ordinárias em um problema de encontrar a solução de um sistema de equações lineares de primeiro grau. Com isso, encontramos a solução mais geral possível da equação de movimento que rege a dinâmica do sistema em termos das componentes dos vetores $\vec{B}, \vec{E}$ e $\vec{v}(0)$ onde, a partir da solução geral, mostramos como obter a solução de três casos particulares cuja importância e aplicabilidade excedem o ponto de vista didático. Por fim, motivamos o nosso estudo fazendo o tratamento quântico do sistema estudado, onde consideramos um feixe de partículas sem spin carregadas. Nesse contexto, mostramos que a curva parametrizada do valor esperado (em média) do vetor posição de uma partícula do feixe em função do tempo corresponde aquela curva associada ao seu análogo clássico.

A consideração de campos $\vec{B}$ e $\vec{E}$ como foi feito aqui nos permite mostrar que a dinâmica do sistema é dada por um sistema de equações diferenciais acopladas com coeficientes constantes. No entanto, algumas outras situações não foram discutidas, como o caso de campos não estáticos nem homogêneos, de modo que alguns fenômenos não foram levados em consideração, como o potencial retardado criado pela corrente elétrica devido ao movimento da carga 16. Além disso, existem uma gama de dinâmicas cuja solução pode ser analisada por meio de transformadas integrais e que são de interesse mais amplo, como a solução da Eq. de Lindblad [17] que descreve a dinâmica de sistemas quânticos sob influências do ambiente (decoerência). Todas essas situações não discutidas aqui serão as motivações de nossos desenvolvimentos futuros, uma vez que a dinâmica agora deve passar a ser regida por um sistema de equações diferenciais acopladas com maior número de equações e onde podemos ter coeficientes de acoplamento dependentes do tempo e/ou posição.

\section{Agradecimentos}

A.C.S. gostaria de agradecer ao Conselho Nacional de Desenvolvimento Científico e Tecnológico (CNPq) e ao Instituto Nacional de Ciência e Tec- 
nologia de Informação Quântica (INCT-IQ) pelo suporte financeiro a esse projeto. M.L.M. agradece ao departamento de Física da Universidade Regional do Cariri - URCA.

\section{Referências}

[1] A. Sommerfeld, Lectures on Theoretical Physics, vol. 1 (Academic Press Inc, New York, 1952), 3th ed.

[2] L.D. Landau and E.M. Lifchitz, Course of Theoretical Physics, vol. 1 (Elsevier, New York, 1976), 3th ed.

[3] D.J. Griffiths, Introduction to Electrodynamics (Prentice Hall, New Jersey, 1999), 3th ed.

[4] H.M. Nusseszveig, Curso de Física Básica, vol. 3, (Blücher, São Paulo, 1997), $1^{\mathrm{a}}$ ed.

[5] S.T. Thornton and J.B. Marion, Classical Dynamics of Particles and Systems (Thomson Learning, Belmont, 2004), 5th ed.

[6] H.M. Nusseszveig, op. cit., p. 129.

[7] N. Riahi, e-print: arXiv:1606.07713 (2016).

[8] B.U. Felderhof, Journal of Physics A 41, 445302 (2008).

[9] G.G. Calderon and A. Rubio, Phys. Rev. A 55, 3370 (1997).

[10] G.B. Arfken and H.J. Weber, Física Matemática

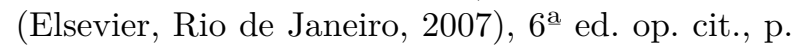
736.

[11] R.L. Viana, Introdução à Eletrodinâmica Clássica (Universidade Federal do Paraná, Curitiba, 2012), p. 209.

[12] C.S. Pereira, Processo de Attachment, Densidade de Raios e a Ocorrência de Flashover em Linhas de Transmissão de Eletricidade (Universidade Federal do Pará, Belém, 2014), p. 98.

[13] P. Ehrenfest, Zeitschrift für Physik 45, 445 (1927).

[14] A.O. Bolivar, Rev. Bras. Ensino Fís. 23, 190 (2001).

[15] J.J. Sakuray, Modern Quantum Mechanics (AddisonWesley, Boston, 1995).

[16] J.D. Jackson, Classical Electrodynamics, (John Wiley and Sons, New York, 1962), 3th ed.

[17] M.A. Nielsen and I.L. Chuang, Quantum Computation and Quantum Information (Cambridge University Press, Cambridge, 2000). 